Commun. math. Phys. 20, 193-204 (1971)

(C) by Springer-Verlag 1971

\title{
Time Development of Quantum Lattice Systems
}

\author{
Mary Beth RuskaI * \\ Institut de Physique Théorique, Université de Genève, Genève, Suisse
}

Received September 20, 1970

\begin{abstract}
The time development of quantum lattice systems is studied with a weaker assumption on the growth of the potential than has been considered previously.
\end{abstract}

\section{Introduction}

The problem of describing the time development of a statistical mechanical system has not yet been treated satisfactorily. In the algebraic approach to statistical mechanics, it has often been assumed that timetranslations correspond to automorphisms of the algebra of quasi-local observables [1]. This assumption has been justified in a few very special cases [2-4], but is not true in general. In particular, it has been shown to be invalid for the ideal Bose gas and BCS models [5]. Indeed, it would be rather surprising if such an assumption were generally valid because it would imply that even those states which are not physically realizable have a well-behaved time development. Therefore, it would seem desirable to study the time-development in a simple case without this assumption. In this paper we consider the time-development of a quantum lattice system. Our assumptions about the growth of the potential are less restrictive than those of Robinson [2], which imply that timetranslations correspond to automorphisms of the algebra.

A lattice system is one which is parametrized so that it can be identified with $\boldsymbol{Z}^{v}$, the space of $v$-tuples of integers. A Hilbert space, $\mathscr{H}(x)$, of finite dimension, $N$, is associated with each lattice site $x$ in $\boldsymbol{Z}^{v}$. The Hilbert space

$$
\mathscr{H}(\Lambda)=\bigotimes_{x \in \Lambda} \mathscr{H}(x)
$$

is associated with each finite region $\Lambda$ in $Z^{v}$. The algebra of local observables for $\Lambda, \mathfrak{A}(\Lambda)$, is simply the algebra of bounded operators on $\mathscr{H}(\Lambda)$. If $\Lambda_{1} \subset \Lambda_{2}$, one can identify every $A$ in $\mathfrak{A}\left(\Lambda_{1}\right)$ with the operator $A \otimes I_{\Lambda_{2} \backslash \Lambda_{1}}$ in $\mathfrak{U}\left(\Lambda_{2}\right)$, where $I_{\Lambda_{2} \backslash \Lambda_{1}}$ is the identity on $\mathscr{H}\left(\Lambda_{2} \backslash \Lambda_{1}\right)$. Then one

\footnotetext{
$\star$ Battelle Fellow.
} 
can define the algebra of quasi-local observables, $\mathfrak{A}$, as the norm closure of

$$
\bigcup_{\Lambda \subset \mathbf{Z}^{v}} \mathfrak{A}(\Lambda) \text {. }
$$

$\mathfrak{A}$ is a $C^{*}$-algebra. Translations of the lattice correspond to commuting automorphisms $g_{x}$ of $\mathfrak{I}$. The Hamiltonian, $H(\Lambda)$, associated with the finite region $\Lambda$ is a self-adjoint operator on $\mathscr{H}(\Lambda)$ which can be written in terms of a potential $\Phi$ as:

$$
H(\Lambda)=\sum_{X \subset A} \Phi(X)
$$

where $\Phi(X)$ is a translation-invariant ${ }^{1}$ self-adjoint operator on $\mathscr{H}(X)$ satisfying $\Phi(\phi)=0$.

We say that a sequence, $\left(\Lambda_{n}\right)$, of finite regions tends to $\infty$ if for every finite $\Delta \subset \boldsymbol{Z}^{v}$ there exists an $N$ such that $n \geqq N$ implies $\Lambda_{n} \supset \Delta$.

The time-development of lattice systems seems to be closely related to the rate at which the potential grows as $X$ increases. For example, suppose that there exists a $\xi>0$ such that

$$
\|\Phi\|_{\xi}=\sum_{X \ni 0}\|\Phi(X)\| \mathrm{e}^{\zeta N(X)}<\infty
$$

where $N(X)$ is the number of lattice sites in $X$. Then it can be shown $[6,7]$ that

$$
\lim _{\Lambda \rightarrow \infty} e^{i t H(\Lambda)} A e^{-i t H(A)}=g_{t} A
$$

exists for all real $t$ and local $A$ in $\mathfrak{U}$, and defines a strongly continuous one-parameter group of $*$-automorphisms of $\mathfrak{A}$. We will consider potentials which satisfy the weaker condition

$$
\|\Phi\|=\sum_{X \ni 0}\|\Phi(X)\|<\infty .
$$

A state on $\mathfrak{A}(\Lambda)$ or $\mathfrak{A}$ is a positive linear functional, $f$, which is normalized so that

$$
f(I)=1
$$

where $I$ is the identity. We will be particularly interested in the Gibbs states which are defined by

for all $A$ in $\mathfrak{U}(\Lambda)$.

$$
\varrho^{\Lambda}(A)=\frac{\operatorname{Tr}\left(e^{-H(A)} A\right)}{\operatorname{Tr}\left(e^{-H(A)}\right)}
$$

\footnotetext{
${ }^{1}$ See [6], p. 15.
} 


\section{Time Evolution of the Gibbs State}

First consider the time-development of the system in a finite region 1. Let

$$
A^{\Lambda}(\xi)=e^{-\xi H(A)} A e^{\xi H(A)}
$$

where $A$ is in $\mathfrak{A}(\Lambda)$,

and

$$
\xi \text { is a complex number, }
$$

$$
H(\Lambda) \text { is given by (1). }
$$

Since $H(\Lambda)$ is bounded, $A^{\Lambda}(\xi)$ is in $\mathfrak{A}(\Lambda)$. Thus, when $\xi=-i$, (4) defines an automorphism of $\mathfrak{A}(\Lambda)$ which describes the time development of the system in the region $\Lambda$.

Now when $A \rightarrow \infty$, (4) is not necessarily well-defined. Therefore, we consider instead the expectation values of products of operators in the Gibbs states, (3). That is, we consider the limit as $\Lambda_{n} \rightarrow \infty$ of

$$
\begin{aligned}
& Q^{A_{n}}\left(A_{1}\left(\xi_{1}\right) \ldots A_{k}\left(\xi_{k}\right)\right) \\
& \quad=\frac{\operatorname{Tr}\left(e^{-H(\Lambda)} e^{-\check{\zeta}_{1} H(A)} A_{1} e^{\check{\zeta}_{1} H(A)} \ldots e^{-\check{\zeta ̆}_{k} H(A)} A_{k} e^{\xi_{k} H(A)}\right)}{\operatorname{Tr}\left(e^{-H(A)}\right)}
\end{aligned}
$$

in the region:

$$
\mathscr{D}_{k}=\left\{\left(\xi_{1} \ldots \xi_{k}\right): \beta_{1}<\beta_{2}<\cdots<\beta_{k}<\beta_{1}+1\right\}
$$

where $\xi_{j}=\beta_{j}-i t_{j}$.

The distinguished boundary of $\mathscr{D}_{k}$ is the set

$$
\begin{aligned}
e\left(\mathscr{D}_{k}\right)= & \left\{\left(\xi_{1} \ldots \xi_{k}\right): \beta_{1}=\beta_{2}=\beta_{j}<\beta_{j+1}=\beta_{k}=\beta_{1}+1\right. \\
& \text { for some } j, \quad 1 \leqq j \leqq k\} .
\end{aligned}
$$

The following theorem shows that (5) has well-defined limits when $\Lambda_{n} \rightarrow \infty$.

Theorem 1. Let $\varrho^{\Lambda_{n}}$ and $\mathscr{D}_{k}$ be defined by (5) and (6) respectively, and let $\left(\Lambda_{n}\right)$ be any sequence $\rightarrow \infty$. Then there exists a subsequence $\left(\Omega_{n}\right)$ and, for every $k$ and $A_{1}, \ldots, A_{k}$ in $\mathfrak{A}$, a function $F_{A_{1} \ldots A_{k}}\left(\xi_{1} \ldots \xi_{k}\right)$ such that

a) When $A_{1} \ldots A_{k}$ are local, i.e. in $\mathfrak{U}\left(\Lambda_{0}\right)$ for some finite $\Lambda_{0}$, $Q^{\Omega_{n}}\left(A_{1}\left(\xi_{1}\right) \ldots A\left(\xi_{k}\right)\right)$ converges uniformly on the compact subsets of $\overline{\mathscr{D}}_{k}$ to $F_{A_{1} \ldots A_{k}}\left(\xi_{1} \ldots \xi_{k}\right)$.

b) $F_{A_{1} \ldots A_{k}}$ is analytic in $\mathscr{D}_{k}$.

c) $F_{A_{1} \ldots A_{k}}$ is continuous in $\overline{\mathscr{D}}_{k}$.

d) $\left|F_{A_{1} \ldots A_{k}}\left(\xi_{1} \ldots \xi_{k}\right)\right| \leqq \prod_{i=1}^{k}\left\|A_{i}\right\| \quad$ in $\overline{\mathscr{D}}_{k}$.

e) The expressions $F_{A_{1} \ldots A_{k}}$ are linear separately in each $A_{i}$. The last two conditions imply that when $B_{i}^{l} \rightarrow A_{i}$ in norm then $F_{B_{1}^{l} \ldots B_{k}^{l}} \rightarrow F_{A_{1} \ldots A_{k}}$ uniformly in $\xi_{1} \ldots \xi_{k}$. 
Before proving Theorem 1, we consider some properties of $\varrho^{4}$.

Theorem 2. For each choice of $k, A_{1}, \ldots A_{k}$ consider $\varrho^{\Lambda}\left(A_{1}\left(\xi_{1}\right) \ldots A_{k}\left(\xi_{k}\right)\right)$ as a function $f\left(\xi_{1} \ldots \xi_{k}\right)$ of $k$ complex variables. Then

a) $f$ is analytic in $\mathscr{D}_{k}$.

b) $f$ is continuous and bounded in $\overline{\mathscr{D}}_{k}$.

c) $\left|f\left(\xi_{1} \ldots \xi_{k}\right)\right| \leqq \prod_{i=1}^{k}\left\|A_{i}\right\| \quad$ in $\overline{\mathscr{D}}_{k}$.

d) $\left|\frac{\partial}{\partial \xi_{j}} f\left(\xi_{1} \ldots \xi_{k}\right)\right| \leqq 2 N\left(\Lambda_{0}\right)\|\Phi\| \prod_{i=1}^{k}\left\|A_{i}\right\|$ in $\overline{\mathscr{D}}_{k}$.

where $\mathscr{D}_{k}$ is defined by (6), $\|\Phi\|$ is defined by (2), and $\Lambda_{0}$ is a finite region chosen so that $A_{1} \ldots A_{k}$ are in $\mathfrak{U}\left(\Lambda_{0}\right)$.

Note that the bounds in (c) and (d) are independent of $\Lambda$.

Proof. It is easy to see that $f\left(\xi_{1} \ldots \xi_{k}\right)$ is actually an entire function and remains bounded on $\mathscr{D}_{k}$, so that (a) and (b) are satisfied. To prove (c), we first show that it is sufficient to consider the maximum of $\left|f\left(\xi_{1} \ldots \xi_{k}\right)\right|$ on $e\left(\mathscr{D}_{k}\right)$. To do this, we exploit the fact that $f$ depends only on differences and make the change of variables.

$$
\omega_{j}=\xi_{j+1}-\xi_{j}=\alpha_{j}+i \eta_{j} \quad(j=1 \ldots k-1) .
$$

Then $\alpha_{j}>0$ and $\sum_{j} \alpha_{j}<1$. Now by fixing $k-2$ of the $\omega_{j}\left(j \neq j_{0}\right)$ one can apply the usual principle of the maximum ${ }^{2}$ to $f$ considered as an analytic function of one variable in the region

$$
0<\alpha_{j_{0}}<1-\sum_{j \neq j_{0}} \alpha_{j}
$$

to show that it approaches its maximum modulus when $\alpha_{j_{0}}=0$ or $\sum_{j} \alpha_{j}=1$. Then since all but one $\omega_{j}$ were arbitrary, $f\left(\xi_{1} \ldots \xi_{k}\right)$ approaches its maximum when either

or

$$
\beta_{1}=\beta_{2}=\cdots=\beta_{k} \quad\left(\text { all } \alpha_{j}=0\right)
$$

$$
\beta_{k}=\beta_{1}+1 \quad\left(\sum_{j} \alpha_{j}=1\right) .
$$

In the latter case one can repeat the argument by considering $\left.f\left(\xi_{1} \ldots \xi_{k}\right)\right|_{\xi_{k}=\xi_{1}+1}$ as a function of $k-1$ variables. Continuing this process, one finds that at each step $|f|$ approaches its maximum either when

$$
\beta_{1}=\beta_{2}=\cdots=\beta_{j}
$$

${ }^{2}$ If a function is holomorphic and bounded on a strip, then the supremum of its modulus on the strip is equal to the supremum of its modulus on the boundary. 
under the assumption $\beta_{j+1}=\cdots=\beta_{k}=\beta_{1}+1$, or when $\beta_{j}=\beta_{j+1}$ $=\cdots=\beta_{k}=\beta_{1}+1$.

Thus $f\left(\xi_{1} \ldots \xi_{k}\right)$ approaches its maximum on $e\left(\mathscr{D}_{k}\right)$. To estimate $|f|$ there we use the formula

$$
\frac{\operatorname{Tr}(B C)}{\operatorname{Tr} B} \leqq\|C\|
$$

which is valid on finite dimensional Hilbert spaces whenever $B$ is a positive operator. On $e\left(\mathscr{D}_{k}\right)$ one has

$$
f=\frac{\operatorname{Tr}\left[A_{1}\left(i t_{1}\right) \ldots A_{j}\left(i t_{j}\right) e^{-H(1)} A_{j+1}\left(i t_{j+1}\right) \ldots A_{k}\left(i t_{k}\right)\right]}{\operatorname{Tr}\left[e^{-H(A)}\right]} .
$$

Then using (8), cyclicity of the trace, and the properties of the norm, one immediately gets

$$
\left|f\left(\xi_{1} \ldots \xi_{k}\right)\right| \leqq\left\|A_{1}\right\| \ldots\left\|A_{k}\right\|,
$$

which proves (c). To prove (d) note that

$\frac{\partial}{\partial \xi_{j}} f=\frac{\operatorname{Tr}\left(e^{-H(\Lambda)} A_{1}\left(\xi_{1}\right) \ldots e^{-\xi_{j} H(\Lambda)}\left[-H(\Lambda), A_{j}\right] e^{\xi_{j} H(\Lambda)} \ldots A_{k}\left(\xi_{k}\right)\right)}{\operatorname{Tr}\left(e^{-H(\Lambda)}\right)}$.

Proceeding as in the proof of $(c)$ one gets

Now

$$
\left|\frac{\partial}{\partial \xi_{j}} f\right| \leqq \prod_{i \neq j}\left\|A_{i}\right\|\left\|\left[H(\Lambda), A_{j}\right]\right\| \text {. }
$$

Therefore

$$
\begin{aligned}
\|[H(A), A]\| & \left.=\| \sum_{\substack{X \subset A \\
X \cap A_{0} \neq \phi}}[\Phi(X), A)\right] \| \\
& \leqq 2\|A\| \sum_{x \in \Lambda_{0}} \sum_{X \ni x}\|\Phi(X)\| \\
& =2\|A\| N\left(\Lambda_{0}\right)\|\Phi\| .
\end{aligned}
$$

$$
\left|\frac{\partial}{\partial \xi_{j}} f\right| \leqq 2 N\left(\Lambda_{0}\right)\|\Phi\| \prod_{i=1}^{k}\left\|A_{i}\right\|
$$

which proves $(\mathrm{d})$.

We now prove Theorem 1.

Proof. Let $A_{1} \ldots A_{k}$ be in $\mathfrak{U}\left(A_{0}\right)$ and let $f_{n}$ be the analytic function corresponding to $\varrho^{\Lambda_{n}}\left(A_{1}\left(\xi_{1}\right) \ldots A_{k}\left(\xi_{k}\right)\right)$ as in Theorem 2 . Then the functions $f_{n}$ and their derivatives are bounded uniformly in $n$. Therefore the sequence $\left(f_{n}\right)$ is equicontinuous and one can apply the Arzelà-Ascoli Theorem $^{3}$. Thus for each choice of $k, A_{1}, \ldots A_{k},\left(\Lambda_{n}\right)$ has a subsequence

\footnotetext{
${ }^{3}$ See Appendix.
} 
$\left(\Lambda_{n^{\prime}}\right)$ such that $\left(f_{n^{\prime}}\right)$ converges on every compact subret of $\overline{\mathscr{D}}_{k}$ to a continuous function $F_{A_{1} \ldots A_{k}}\left(\xi_{1} \ldots \xi_{k}\right)$. We want to show that this subsequence can be chosen independently of $k, A_{1} \ldots A_{k}$. Let $X$ be a countable, dense subset of $\mathfrak{A}$. Then

$$
\bigcup_{k=1}^{\infty}\left\{B_{1} \ldots B_{k}: B_{i} \text { in } X\right\}
$$

is countable. By the usual Cantor diagonalization procedure one can find a subsequence $\left(\Omega_{n}\right)$ of $\left(\Lambda_{n}\right)$ such that $\varrho^{\Omega_{n}}\left(B_{1}\left(\xi_{1}\right) \ldots B_{k}\left(\xi_{k}\right)\right)$ converges for all $k$ and $B_{1} \ldots B_{k}$ in $X$. Now let $A_{1} \ldots A_{k}$ be arbitrary local elements of $\mathfrak{A}$ and choose $B_{i}$ in the dense set $X$ so that

$$
\left\|A_{j}-B_{j}\right\| \leqq \varepsilon\left[(2 k+1) \prod_{i=1}^{j-1}\left\|B_{i}\right\| \prod_{i=j+1}^{k}\left\|A_{i}\right\|\right]^{-1} .
$$

Since $\varrho^{\Omega_{n}}$ converges on $X \otimes X \otimes \cdots \otimes X$ one can find an $N$ such that $n, m \geqq N$ implies

$$
\left|\varrho^{\Omega_{n}}\left(B_{1}\left(\xi_{1}\right) \ldots B_{k}\left(\xi_{k}\right)\right)-\varrho^{\Omega_{m}}\left(B_{1}\left(\xi_{1}\right) \ldots B_{k}\left(\xi_{k}\right)\right)\right|<\frac{\varepsilon}{2 k+1} .
$$

Then $n, m \geqq N$ implies that

$$
\begin{aligned}
\mid \varrho^{\Omega_{n}}\left(A_{1}\left(\xi_{1}\right) \ldots A_{k}\left(\xi_{k}\right)\right)-\varrho^{\Omega_{m}}\left(A_{1}\left(\xi_{1}\right)\right. & \left.\ldots A_{k}\left(\xi_{k}\right)\right) \mid \\
= & \mid \varrho^{\Omega_{n}}\left(\left(A_{1}-B_{1}\right)\left(\xi_{1}\right) A_{2}\left(\xi_{2}\right) \ldots A_{k}\left(\xi_{k}\right)\right) \\
& +\varrho^{\Omega_{n}}\left(B_{1}\left(\xi_{1}\right)\left(A_{2}-B_{2}\right)\left(\xi_{2}\right) \ldots A_{k}\left(\xi_{k}\right)\right) \\
& +\cdots+\varrho^{\Omega_{n}}\left(B_{1}\left(\xi_{1}\right) \ldots B_{k}\left(\xi_{k}\right)\right) \\
& -\varrho^{\Omega_{m}}\left(B_{1}\left(\xi_{1}\right) \ldots B_{k}\left(\xi_{k}\right)\right) \\
& -\varrho^{\Omega_{m}}\left(B_{1}\left(\xi_{1}\right) \ldots B_{k-1}\left(\xi_{k-1}\right)\left(A_{k}-B_{k}\right)\left(\xi_{k}\right)\right) \\
& -\cdots \varrho^{\Omega_{m}}\left(\left(A_{1}-B_{1}\right)\left(\xi_{1}\right) A_{2}\left(\xi_{2}\right) \ldots A_{k}\left(\xi_{k}\right)\right) \mid
\end{aligned}
$$

$\leqq \varepsilon$ for all $\xi_{1} \ldots \xi_{k}$ in any compact subset of $\overline{\mathscr{D}}_{k}$.

This proves part (a). Since the $\left(\varrho^{\Lambda_{n}}\right)$ are linear in each $A_{i}$ and uniformly bounded, the limit is also linear and bounded by

$$
\left|F_{A_{1} \ldots A_{k}}\left(\xi_{1} \ldots \xi_{k}\right)\right| \leqq \prod_{i=1}^{k}\left\|A_{i}\right\| .
$$

Now let $A_{1} \ldots A_{k}$ be arbitrarily elements in $\mathfrak{Q}$ and let $\left(B_{i}{ }^{n}\right)$ be a sequence of local elements converging to $A_{i}$. Then boundedness and linearity imply that one can choose $N$ so that whenever $n, m \geqq N$

$$
\left|F_{B_{1}^{n}}^{n} \ldots B_{k}^{n}-F_{B_{1}^{m}} \ldots B_{k}^{m}\right|<\varepsilon
$$


for all $\xi_{1} \ldots \xi_{k}$ in $\overline{\mathscr{D}}_{k}$. Therefore the functions $\left(F_{B_{1}^{n}} \ldots B_{k}^{n}\right)$ converge uniformly on compact subsets of $\mathscr{D}_{k}$ to a limit $F_{A_{1} \ldots A_{k}}$ which satisfies, (b), (c), (d), and (e).

The functions $F_{A_{1} \ldots A_{k}}$ are, of course, related to the Gibbs state on $\mathfrak{A}$. In fact one can identify the following quantities with the Gibbs state on $\mathfrak{A}$ :

a) $F_{A}(\xi)=F_{A}(0)$ for all $\xi$.

b) $F_{A_{1} \ldots A_{k}}\left(\xi_{1} \ldots \xi_{k}\right)$ for each fixed $\xi_{1} \ldots \xi_{k}$ such that $\xi_{1} \ldots \xi_{k}$ is in $e\left(\mathscr{D}_{k}\right)$ and $t_{1}=t_{2}=\cdots=t_{k}$.

c) $F_{A_{1} \ldots A_{k}}\left(\xi_{1} \ldots \xi_{k}\right)$ for each fixed $\xi_{1} \ldots \xi_{k}$ when $\lim _{A \rightarrow \infty} A^{\Lambda}(\xi)$ exists for all local $A$ in $\mathfrak{A}$.

The translation invariance of the potential has been used only in the proof of part (d) of Theorem 2. One can actually drop this requirement if (2) is replaced by

$$
\|\Phi\|_{x}=\sum_{X \ni x}\|\Phi(X)\|<\infty \quad \text { for all } x \text { in } \boldsymbol{Z}^{v} .
$$

Non-translationally invariant potentials have been discussed by Brascamp [8].

It is also interesting to note that a result analogous to part (a) of Theorem 1 has been proved for the case of dilute quantum continuous gases [9].

\section{Representation in a Hilbert Space}

We have already noted that the algebra of quasi-local observables at non-zero time, $t$, is not necessarily identical to $\mathfrak{A}$, the algebra at time $t=0$. Therefore we wish to consider a larger algebra, $\mathscr{W}$, defined as the free algebra of polynomials generated by

$$
\left\{\left(A_{i}, t_{i}\right): A_{i} \text { in } \mathfrak{A}, t_{i} \text { in } \boldsymbol{R}\right\} .
$$

Elements of $\mathscr{W}$ are denoted by

$$
\mathscr{P}\left(\left(A_{1} t_{1}\right),\left(A_{2}, t_{2}\right) \ldots\left(A_{k}, t_{k}\right)\right)=\mathscr{P}\left(A_{i}, t_{i}\right),
$$

and conjugation is defined by

$$
\mathscr{P}^{*}\left(\left(A_{1}, t_{1}\right) \ldots\left(A_{k}, t_{k}\right)\right)=\mathscr{P}\left(\left(A_{k}^{*}, t_{k}\right) \ldots\left(A_{1}^{*}, t_{1}\right)\right) .
$$

$\mathscr{W}$ is a $*$-algebra, but not a $C^{*}$-algebra. Indeed, we have not even defined a norm on all of $\mathscr{W}$. Nevertheless, we will show that one can construct a representation of $\mathscr{W}$ on the algebra of bounded operators of some Hilbert space. This construction is completely analogous to the usual Gelfand-Naimark-Segal (GNS) representation [10] for $C^{*}$-algebras. The 
only difference is that the GNS construction uses the completeness of the algebra to prove that the image operators are bounded, while we must use special properties of the Gibbs states to show this ${ }^{4}$.

We now define a functional on $\mathscr{W}$ which can be used to implement the construction of the GNS Hilbert space. Let

$$
\sigma\left(\left(A_{1}, t_{1}\right) \ldots\left(A_{k}, t_{k}\right)\right)=F_{A_{1} \ldots A_{k}}\left(-i t_{1} \ldots-i t_{k}\right)
$$

and extend $\sigma$ to all of $\mathscr{W}$ by linearity. Parts (a) and (b) of the following theorem show that $\sigma$ is the analogue of a state on $\mathscr{W}$.

Theorem 3. Let $\mathscr{W}$ and $\sigma$ be the free algebra and functional defined in (13) and (15). Then $\sigma$ has the following properties:

a) $\sigma$ is positive, i.e. $\sigma(\mathscr{P} * \mathscr{P}) \geqq 0$ for all $\mathscr{P}$ in $\mathscr{W}$.

b) $\sigma(I, t)=1$ where $I$ is the identity in $\mathscr{W}$ and $t$ is in $\boldsymbol{R}$.

c) $\left|\sigma\left(\mathscr{P}_{1}^{*},(A, t), \mathscr{P}_{2}\right)\right| \leqq\|A\|\left[\sigma\left(\mathscr{P}_{1} * \mathscr{P}_{1}\right) \sigma\left(\mathscr{P}_{2} * \mathscr{P}_{2}\right)\right]^{\frac{1}{2}}$.

d) $\sigma\left(\left(A_{1}, t_{1}\right) \ldots\left(A_{k}, t_{k}\right)\right)=\sigma\left(\left(A_{1}, t_{1}+\tau\right) \ldots\left(A_{k}, t_{k}+\tau\right)\right)$.

Proof. To prove (a) consider only those $\mathscr{P}\left(A_{i} t_{i}\right)$ for which all $A_{i}$ are local, i.e. in some $\mathfrak{A}\left(\Lambda_{0}\right)$. Then

$$
\sigma(\mathscr{P} * \mathscr{P})=\lim _{n \rightarrow \infty} \operatorname{Tr}\left(B_{n}{ }^{*} B_{n}\right) / \operatorname{Tr}\left(C_{n}{ }^{*} C_{n}\right)
$$

where

$$
\begin{aligned}
& B_{n}=\mathscr{P}\left(A_{i}\left(-i t_{i}\right)\right) e^{-H\left(\Omega_{n}\right) / 2} \\
& C_{n}=e^{-H\left(\Omega_{n}\right) / 2} .
\end{aligned}
$$

Then part (e) of Theorem 1 implies that $\sigma$ is positive for all $\mathscr{P}$. Parts (b) and (d) are trivial. To prove (c) we again need to consider only local $A_{i}$. Let $\pi^{n}(A)$ be the image of $A$ in the usual GNS representation of $\mathfrak{A}\left(\Omega_{n}\right)$ defined by $\varrho^{\Omega_{n}}$. Then

$$
\begin{aligned}
\left|\sigma\left[\mathscr{P}_{1}^{*}\left(A_{j}, t_{j}\right)(B, \tau) \mathscr{P}_{2}\left(A_{j}, t_{j}\right)\right]\right|^{2} \\
\quad=\lim _{n \rightarrow \infty}\left|\varrho^{\Omega_{n}}\left[\mathscr{P}_{1} *\left(A_{j}\left(-i t_{j}\right)\right) B(-i \tau) \mathscr{P}_{2}\left(A_{j}\left(-i t_{j}\right)\right)\right]\right|^{2} \\
\quad \leqq \lim _{n \rightarrow \infty}\left[\varrho^{\Omega_{n}}\left(\mathscr{P}_{1} * \mathscr{P}_{1}\right) \varrho^{\Omega_{n}}\left(\mathscr{P}_{2} * \mathscr{P}_{2}\right)\left\|\pi^{n}(B(-i \tau))\right\|^{2}\right] \\
\quad \leqq \sigma\left(\mathscr{P}_{1} * \mathscr{P}_{1}\right) \sigma\left(\mathscr{P}_{2} * \mathscr{P}_{2}\right)\|B\|^{2} .
\end{aligned}
$$

We now sketch the construction of our representation of $\mathscr{W}$. Details are similar to those of GNS [10] or Wightman [11]. Let

$$
\Gamma=\left\{\mathscr{P} \in \mathscr{W}: \sigma\left(\mathscr{P}^{*} \mathscr{P}\right)=0\right\} .
$$

$\Gamma$ is a left ideal in $\mathscr{W}$. Let $\tilde{\mathscr{H}}=\mathscr{W} / \Gamma$ be the quotient space of $\mathscr{W}$ with $\Gamma$ and define

$$
\left\langle\psi\left(\mathscr{P}_{1}\right), \psi\left(\mathscr{P}_{2}\right)\right\rangle=\sigma\left(\mathscr{P}_{1} * \mathscr{P}_{2}\right) .
$$

\footnotetext{
${ }^{4}$ A similar representation of field operators has been given by Wightman [11].
} 
Then (16) defines a positive definite inner product on $\tilde{\mathscr{H}}$ and one can complete $\tilde{\mathscr{H}}$ to a Hilbert space $\mathscr{H}$. Define a $*$-representation of $\mathscr{W}$ in $\mathscr{H}$ by

$$
\pi\left(\mathscr{P}_{1}\right) \psi\left(\mathscr{P}_{2}\right)=\psi\left(\mathscr{P}_{1} \mathscr{P}_{2}\right) .
$$

Part (c) of Theorem 3 implies that $\pi(\mathscr{P})$ is a bounded linear operator on $\mathscr{H}$ since

$$
\begin{aligned}
\|\pi(B(t))\| & =\sup _{\mathscr{P}_{1} \mathscr{P}_{2} \notin \Gamma} \frac{\left|\left\langle\psi\left(\mathscr{P}_{1}\right), \pi(B(t)) \psi\left(\mathscr{P}_{2}\right)\right\rangle\right|}{\left\|\psi\left(\mathscr{P}_{1}\right)\right\|\left\|\psi\left(\mathscr{P}_{2}\right)\right\|} \\
& =\sup _{\mathscr{P}_{1} \mathscr{P}_{2} \notin \Gamma} \frac{\left|\sigma\left(\mathscr{P}_{1}^{*},(B, t), \mathscr{P}_{2}\right)\right|}{\left[\sigma\left(\mathscr{P}_{1}^{*} \mathscr{P}_{1}\right) \sigma\left(\mathscr{P}_{2} * \mathscr{P}_{2}\right)\right]^{\frac{1}{2}}} \\
& \leqq\|B\| .
\end{aligned}
$$

If $I$ is the identity in $\mathscr{W}$, then $\alpha=\psi(I)$ is a cyclic vector for $\pi$. One can summarize these results in the following theorem:

Theorem 4. Let $\sigma$ be a positive linear functional on $\mathscr{W}$ satisfying conditions (a) and (c) of Theorem 3. Then there exists a $*$-representation, $\pi$, of $\mathscr{W}$ in the bounded operators of some Hilbert space, $\mathscr{H}$, and a cyclic vector, $\alpha$ in $\mathscr{H}$ such that:

a) $\sigma(\mathscr{P})=\langle\alpha, \pi(\mathscr{P}) \alpha\rangle$.

b) $\{\pi(\mathscr{P}) \alpha: \mathscr{P}$ in $\mathscr{W}\}$ is dense in $\mathscr{H}$.

c) $\|\pi(A, t)\| \leqq\|A\|$.

$\mathscr{W}$ has been constructed so that time-translations form a oneparameter group, $G=\left\{g_{\tau}\right\}$, of automorphisms of $\mathscr{W}$ with

$$
g_{\tau} \mathscr{P}\left(A_{i}, t_{i}\right)=\mathscr{P}\left(A_{i}, t_{i}+\tau\right) .
$$

Therefore, every appropriately bounded ${ }^{5}$, positive linear functional, $\sigma$, which is invariant under time-translations, i.e. $\sigma\left(g_{\tau} \mathscr{P}\right)=\sigma(\mathscr{P})$, can be used to define a representation of $G$ on a Hilbert space.

Theorem 5. Let $\sigma$ be a linear functional on $\mathscr{W}$ satisfying conditions (a), (c), and (d) of Theorem 3. Let $\mathscr{H}, \pi$, and $\alpha$ be as in Theorem 4. Then there exists a unique continuous unitary representation $U(\tau)$ of $\boldsymbol{R}$ in $\mathscr{H}$ such that:

a) $U(\tau) \alpha=\alpha$.

b) $U(\tau) \pi(\mathscr{P}) U(-\tau)=\pi\left(g_{\tau} \mathscr{P}\right)$.

c) $\sigma\left(\left(A_{1}, t_{1}\right) \ldots\left(A_{k}, t_{k}\right)\right)$ $=\left\langle\alpha, \pi\left(A_{1}\right) U\left(t_{2}-t_{1}\right) \pi\left(A_{2}\right) \ldots U\left(t_{k}-t_{k-1}\right) \pi\left(A_{k}\right), \alpha\right\rangle$.

d) $\left\{\mathscr{P}\left(\pi\left(A_{i}\right), U\left(\tau_{i}\right)\right) \alpha: A_{i}\right.$ in $\mathfrak{A}, \tau_{i}$ in $\boldsymbol{R}, \mathscr{P}$ is a polynomial in $\pi\left(A_{i}\right)$ $=\pi\left(A_{i}, 0\right)$ and $\left.U\left(\tau_{i}\right)\right\}$ is dense in $\mathscr{H}$.

${ }^{5}$ Condition (c) of Theorem 3. 
Proof. The proof of (a) and (b) is identical to that of a similar theorem for $C^{*}$-algebras ${ }^{6}$. We merely note that $U(\tau)$ can be defined by

$$
U(\tau) \pi\left(\mathscr{P}\left(A_{i} t_{i}\right)\right) \alpha=\pi\left(\mathscr{P}\left(A_{i}, t_{i}+\tau\right)\right) \alpha .
$$

Parts (c) and (d) then follow easily from Theorem 4.

\section{Translation Invariance}

The functionals considered so far need not be translation invariant. In this section we show that there exists a translation invariant functional which has the same time-development as $\sigma$, the functional which describes the time-development of the Gibbs states.

First we digress to show that the spaces which we use are compact. Let $\mathscr{E}$ be the space of linear functionals on $\mathscr{W}$ which satisfy

$$
\left|\gamma\left(\left(A_{1}, t_{1}\right) \ldots\left(A_{k}, t_{k}\right)\right)\right| \leqq \prod_{i=1}^{k}\left\|A_{i}\right\|
$$

and put on $\mathscr{E}$ the weak topology. Then $\mathscr{E}$ is homeomorphic to the space $M$, defined by

$$
M=\prod_{\alpha}\left[-\mu_{\alpha}, \mu_{\alpha}\right]
$$

where $\alpha$ labels all possible choices of $k, A_{1}, \ldots A_{k}, t_{1}, \ldots t_{k}$,

$$
\mu_{\alpha}=\prod_{i=1}^{k_{\alpha}}\left\|A_{i_{\alpha}}\right\|
$$

and $M$ has the usual product topology. Then since $M$ is compact, $\mathscr{E}$ is also compact in the weak topology. Thus, any closed subset of $\mathscr{E}$ will be compact.

Now define

$$
\begin{aligned}
g_{x} \sigma\left(\mathscr{P}\left(A_{i}, t_{i}\right)\right) & =\sigma_{x}\left(\mathscr{P}\left(A_{i}, t_{i}\right)\right) \\
& =\sigma\left(\mathscr{P}\left(g_{x} A_{i}, t_{i}\right)\right)
\end{aligned}
$$

where $g_{x} A_{i}$ is defined by the automorphism of $\mathfrak{A}$ corresponding to translation by $x$ in $\boldsymbol{Z}^{\nu}$. Since $\left\|g_{x} A\right\|=\|A\|, \sigma_{x}$ is in $\mathscr{E}$. Let $\mathscr{K}$ be the closed convex hull of $\left\{\sigma_{x}: x \in \boldsymbol{Z}^{v}\right\} . \mathscr{K}$ is a closed, and therefore compact, subset of $\mathscr{E}$.

For every $x$ in $Z^{v}$, define a mapping $g_{x}$ on $\mathscr{K}$ by

$$
g_{x} \sigma_{y}=\sigma_{x+y} .
$$

\footnotetext{
${ }^{6}$ See Ref. [6], p. 147.
} 
$g_{x}$ has the following properties:

a) $g_{x} \mathscr{K}=\mathscr{K}$.

b) $g_{x}$ is commutative, i.e. $g_{x} g_{y}=g_{y} g_{x}$.

c) $g_{x}$ is linear.

d) $g_{x}$ is continuous.

Thus, by the Markov-Kakutani Theorem ${ }^{7}$, there exists a functional, $\hat{\sigma}$, in $\mathscr{K}$ such that

$$
g_{x} \hat{\sigma}=\hat{\sigma} \quad \text { for all } x \text { in } Z^{v},
$$

i.e. $\hat{\sigma}$ is translation invariant.

Since $\sigma$ satisfies the hypotheses of Theorem 3, so do all elements of $\mathscr{K}$. For example, if $\omega=\sum_{i} \lambda_{i} \sigma_{x_{i}}$, then

$$
\begin{aligned}
\mid \omega\left(\mathscr{P}_{1} *\left(A_{j}, t_{j}\right),(B, \tau)\right. & \left.\mathscr{P}_{2}^{*}\left(A_{j}, t_{j}\right)\right) \mid \\
& =\left|\sum_{i} \lambda_{i} \sigma\left[\left(\tau_{x_{l}} \mathscr{P}_{1}\right)^{*}\left(\tau_{x_{l}} B, \tau\right)\left(\tau_{x_{i}} \mathscr{P}_{2}\right)\right]\right| \\
& \leqq \sum_{i} \lambda_{i}\left\{\sigma\left[\left(\tau_{x_{i}} \mathscr{P}_{1}\right)^{*}\left(\tau_{x_{i}} \mathscr{P}_{1}\right)\right] \sigma\left[\left(\tau_{x_{i}} \mathscr{P}_{2}\right)^{*}\left(\tau_{x_{i}} \mathscr{P}_{2}\right)\right]\right\}^{\frac{1}{2}}\left\|\tau_{x_{i}} B\right\| \\
& =\sum_{i}\left[\lambda_{i} \sigma_{x_{l}}\left(\mathscr{P}_{1} * \mathscr{P}_{1}\right)\right]^{\frac{1}{2}}\left[\lambda_{i} \sigma_{x_{l}}\left(\mathscr{P}_{2} * \mathscr{P}_{2}\right)\right]^{\frac{1}{2}}\|B\| \\
& \leqq\left[\sum_{i} \lambda_{i} \sigma_{x_{i}}\left(\mathscr{P}_{1} * \mathscr{P}_{1}\right)\right]^{\frac{1}{2}}\left[\sum_{i} \lambda_{i} \sigma_{x_{i}}\left(\mathscr{P}_{2} * \mathscr{P}_{2}\right)\right]^{\frac{1}{2}}\|B\| \\
& \left.=\mid \omega\left(\mathscr{P}_{1} * \mathscr{P}_{1}\right) \omega\left(\mathscr{P}_{2} * \mathscr{P}_{2}\right)\right]^{\frac{1}{2}}\|B\| .
\end{aligned}
$$

Therefore, $\hat{\sigma}$ defines a representation $(\mathscr{H}, \pi, \alpha)$ of $\mathscr{W}$ according to Theorem 4, and a representation, $U(\tau)$ of the time-translations according to Theorem 5 .

\section{Appendix}

Theorem A.1 (Arzelà-Ascoli, [12]).

Let $\left(f_{k}\right)$ be a pointwise bounded, equicontinuous sequence of realor complex-valued functions on a separable metric space $M$. Then $\left(f_{k}\right)$ has a subsequence which converges to a continuous function $g$ pointwise on $M$ and uniformly on every compact subset of $M$.

Theorem A.2 (Markov-Kakutani, [13]).

Let $K$ be a compact convex subset of a linear topological space $\mathscr{X}$. Let $\mathscr{T}$ be a commuting family of continuous linear mappings which map $K$ into itself. Then there exists a point $p$ in $K$ such that $T p=p$ for all $T$ in $\mathscr{T}$.

Acknowledgement. It is a pleasure to thank Professor D. Ruelle for suggesting this problem and for many helpful discussions. The author is also grateful to Prof. J. M. Jauch and Dr. J.-P. Eckmann for their continued interest and encouragement.

\footnotetext{
${ }^{7}$ See Appendix
} 


\section{References}

1. Haag, R., Hugenholtz, N. M., Winnink, M.: Commun. math. Phys. 5, 215 (1967).

2. Robinson, D. W.: Commun. math. Phys. 7, 337 (1968).

3. Streater, R. F.: Commun. math. Phys. 7, 93 (1968).

4. Hepp, K.: Unpublished.

5. Dubin, D. A., Sewell, G. L.: J. Math. Phys. 11, 2990 (1970).

6. Ruelle, D.: Statistical mechanics. Rigorous results. New York: Benjamin 1969.

7. - Lecture notes, Les Houches Summer School, 1970.

8. Brascamp, H. J.: Commun. math. Phys. 18, 82 (1970).

9. Ruelle, D.: Analyticity of Green's functions of dilute quantum gases, preprint.

10. Gelfand, I., Raikov, D., Shilov, G.: Commutative normed rings, Chapter VIII. New York: Chelsea 1964.

11. Wightman, A. S.: Phys. Rev. 101, 860 (1956).

12. Korevaar, J.: Mathematical methods, p. 244. New York: Academic Press 1968.

13. Dunford, N., Schwarz, J. T.: Linear operators, Vol. I, p. 456. New York: Interscience 1958.

Mary Beth Ruskai

Institut de Physique Théorique

Université de Genève

Genève, Suisse 